\title{
ORIGINAL ARTICLE \\ Exploratory study to suggest the possibility of MMP-8 and MMP-9 serum levels as early markers for remission after traumatic spinal cord injury
}

\author{
A Moghaddam ${ }^{1}$, R Heller ${ }^{1}$, V Daniel ${ }^{2}$, T Swing ${ }^{1}$, M Akbar $^{3}, \mathrm{H}-\mathrm{J} \mathrm{Gerner}^{1}$ and B Biglari ${ }^{4}$
}

Study design: A prospective observational study reporting the correlation between matrix metalloprotein serum levels and remission after traumatic spinal cord injury (SCl).

Objectives: To investigate serum cytokine levels as predictive markers.

Setting: Germany, Rhineland-Palatinate (Rheinland-Pfalz).

Methods: Between 2010 and 2015, data sets from 115 patients (33 female, 82 male) after traumatic SCI were recorded at the BG Trauma Centre Ludwigshafen. We examined the serum levels of Matix metallopraoteinases (MMPs) MMP-2, MMP-8, MMP-9, MMP-10 and MMP-12 over a 12-week period, that is, at admission and 4, 9, $12 \mathrm{~h}, 1$ and 3 days and 1, 2, 4, 8 and 12 weeks after trauma. Following the same match-pair procedure as in our previous studies, we selected 10 patients with $\mathrm{SCl}$ and neurological remission (Group 1) and 10 patients with an initial American Spinal Injury Association (ASIA) A grade and no neurological remission (Group 0). Ten patients with an isolated vertebral fracture without neurological deficits served as a control group (Group C). Our analysis was performed using a Luminex Performance Human High Sensitivity Cytokine Panel. Multivariate logistic regression models were used to examine the predictive value of MMPs with respect to neurological remission vs no neurological remission.

Results: MMP-8 and MMP-9 provided significantly different values. The favoured predictive model allows to differentiate between neurological remission and no neurological remission in $97 \%$ of cases.

Conclusions: The results indicate that further studies with an enlarged collective are warranted in order to investigate current monitoring, prognostic and tracking techniques as well as scoring systems.

Spinal Cord (2017) 55, 8-15; doi:10.1038/sc.2016.104; published online 5 July 2016

\section{INTRODUCTION}

Spinal cord injury (SCI) impairs patients' quality of life greatly and causes immense financial consequences for them and their families. ${ }^{1,2}$ Current studies report an average annual SCI incidence of 40 cases per million in the United States. ${ }^{3}$ Despite substantial research on SCI, no ground breaking step has been made towards understanding the mechanisms of SCI and exploring new therapies. New therapies for reversing neurological deficits are also lacking. ${ }^{2}$ Current treatments such as medications or surgical treatment are limited in their success and results are very poor. ${ }^{4-6}$ Furthermore, most therapeutic strategies lack convincing evidence for their beneficial effects. ${ }^{6,7}$ There exist no valid markers specifying the potential for remission up to this point.

The pathophysiological process is characterised by a primary and a secondary phase of injury. Although the first phase is marked by mechanical trauma, the second phase is more complex due to a variety of pathophysiological processes. ${ }^{2}$ The early inflammatory response involves an initial wave of infiltrating neutrophils, followed by migration of monocytes and macrophages into the injured segment. Each of these inflammatory cells expresses MMPs including MMP-2 (gelatinase A), MMP-8 (neutrophil collagenase), MMP-9 (gelatinase B), MMP-11 (stromelysin-3) and MMP-12 (metalloelastase). ${ }^{8}$ Furthermore, MMP-10 is suspected to have a critical role in controlling tissue remodelling in macrophages and moderating scar tissue formation during wound repair. 9

Currently, there are still few reports focused on diagnostic biomarkers in SCI. ${ }^{10,11}$ In view of devastating consequences of SCI and the poor therapeutic solutions, there is an urgent need for greater efforts to innovate reliable biomarkers for remission after SCI. ${ }^{11}$ Because of their relevance in processes after traumatic injuries such as traumatic spinal cord injuries, as well as differing catalytic mechanisms, we decided to investigate MMP-2, MMP-8, MMP-9, MMP-10 and MMP-12.8,9,12 Our goal was to discover a prognostic biomarker for remission potential after SCI by investigating matrixmetalloproteines in peripheral serum and therewith determine neurological outcome. Animal studies investigating possible markers are limited in terms of transferability. ${ }^{13}$ The human model in this study investigated the following: first, if markers can be found in serum to predict rehabilitation post SCI; second, if there is an effective method

${ }^{1}$ HTRG Heidelberg Trauma Research Group, Center for Orthopedics, Trauma Surgery and Spinal Cord Injury, Heidelberg University Hospital, Heidelberg, Germany; ${ }^{2}$ Transplantation Immunology, Institute of Immunology, University of Heidelberg, Heidelberg, Germany; ${ }^{3}$ Spine Center, Center for Orthopedics, Trauma Surgery and Spinal Cord Injury, Heidelberg University Hospital, Heidelberg, Germany and ${ }^{4}$ Department of Paraplegiology, Berufsgenossenschaftliche Unfallklinik Ludwigshafen, Ludwigshafen, Germany Correspondence: Dr Professor Arash Moghaddam, HTRG Heidelberg Trauma Research Group, Center for Orthopedics, Trauma Surgery and Spinal Cord Injury, Heidelberg University Hospital, Schlierbacher Landstraße 200a, Heidelberg 69118, Germany.

E-mail: arash.moghaddam@med.uni-heidelberg.de or email@arash.de

Received 7 April 2016; revised 2 May 2016; accepted 9 May 2016; published online 5 July 2016 
of monitoring neurological remission for future treatment strategies for SCI; third, if results can be used to generate an improved animal model for investigating SCI. ${ }^{14}$

The answers to these questions will help us find a way to monitor improvement, as well as help us develop a useful objective score.

\section{MATERIALS AND METHODS}

Between 2010 and 2015, data sets of 115 patients (33 females and 82 males) were recorded after traumatic SCI in the Berufsgenossenschaftliche Unfallklinik Ludwigshafen (BG Trauma Centre). Following the same match-pair procedure as in our previous studies, ${ }^{15}$ we selected 10 patients with SCI and neurological remission (Group 1=G1) and 10 patients with an initial American Spinal Injury Association (ASIA) A grade and no neurological remission (Group $0=\mathrm{G} 0$ ). Furthermore, we chose 10 patients with an isolated vertebral fracture without neurological deficits to serve as a control group (Group $\mathrm{C}=\mathrm{C})$. Blood was drawn at the same time points in the studies for both groups. Four vials of serum (each $7.5 \mathrm{ml}$ ) were obtained with a standard procedure at different time points, that is, 4, 9, $12 \mathrm{~h}, 1$ and 3 days and 1, 2, 4, 8 and 12 weeks after SCI. After $20 \mathrm{~min}$ of coagulation, blood was centrifuged at 3000 r.p.m., aliquoted and stored at $-80^{\circ} \mathrm{C}$ until analysis. Serum samples were obtained at the same time points in both groups. The method of sample collection is the same as in our previous cytokine analysis. ${ }^{16-18}$ The ASIA impairment scale (AIS) grades were conducted in awake and responsive patients at the time of admission and 12 weeks according to the International Standards for Neurological Classification of SCI (ISNCSCI; Table 1). ${ }^{19,20}$ Because BG Ludwigshafen is a primary trauma centre with its own helicopter patients included in the study were admitted within $2 \mathrm{~h}$ after trauma. ${ }^{16}$ Surgical stabilisation and decompression was carried out $3.52 \pm 1.35 \mathrm{~h}$ after trauma. Quantification was carried out in accordance with the GLP (good laboratory practice) provisions. A clinical examination of the patients took place parallel to blood sampling. Therefore, the recovery process was determined by the parameters of the clinical course (ASIA score). The quantitative measurement of MMP-2, MMP-8, MMP-9, MMP-10 and MMP-12 from patient serum was conducted using the Luminex Performance Human High Sensitivity Cytokine Panel according to the manufacturer's instructions (Catalogue Number FCST07-05, Kit Lot Number 1415263). The kits were provided by R\&D Systems (Minneapolis, MN, USA). The lab technician performing the test was blinded to all patients and clinical information and all lab work was carried out in the Heidelberg University Hospital. ${ }^{16}$ Storage took place until analysis at $-80^{\circ} \mathrm{C}$. Our prospective observational study has been approved by the ethics committee of the University of Heidelberg (S-514/2011) and the Landesärztekammer Rheinland-Pfalz (837.188.12 / 8289-F), Germany. All study participants signed and dated consent forms willingly and could voluntarily choose to leave the study at any time and for any reason. Exclusion criteria were the following: non-traumatic SCI, traumatic brain injury, severe abdominal trauma, traumatic amputation of extremities and coma and all patients with an additional major trauma apart from the SCI. Participants were not given methylprednisolone sodium succinate during study participation.

Table 1 ASIA impairment scale (AIS) grade and functional impairment (clinical state) due to $\mathrm{SCl}$

\section{AIS grade Clinical state}

A Complete-no motor or sensory function is preserved in the sacral segments S4-S5

B Incomplete - sensory but not motor function is preserved below the NLI and includes the sacral segments S4-S5

C Incomplete-motor function is preserved below the $\mathrm{NLI}$, and more than half of the key muscles below the $\mathrm{NLI}$ have a muscle grade $<3$

D Incomplete-motor function is preserved below the NLI, and at least half of the key muscles below the NLI have a muscle grade of 3 or more

\section{Matching}

We compared three groups of patients as in our previous studies: ${ }^{15}$

Patients with traumatic SCI without neurological remission (Group $0=\mathrm{G} 0$ )

Patients with traumatic SCI with neurological remission (Group 1=G1)

Fracture patients without neurological impairment (Control Group $=\mathrm{C}$ )

Patients with traumatic SCI who showed no neurological remission within 3 months were assigned to G0. Those with traumatic SCI who showed neurological remission within 3 months were assigned to G1. We matched the two groups with a third group of patients with vertebral fractures that presented no neurological impairment. Patients were enroled in Group C and served as a control group. Patients were matched on the basis of four criteria: age, sex, aetiology and $\mathrm{AO}$ classification. If more than one match was found for a nonremission, then patients with the most similar clinical profile were chosen and vice versa. According to matching criteria, three groups (each group $n=10$ ) could be formed of the above-mentioned total of 115 study patients (Table 1).

\section{Statistical analysis}

All statistical calculations were performed either with SPSS (SPSS Statistics for Windows 2012, version 21.0, IBM Corp, Armonk, NY, USA) or R version 3.2.3 $3^{21}$ using the package ' $\mathrm{pROC}$ '22 for receiver operator characteristics (ROC) analysis. Figures were created by using GraphPad Prism version 5.00 for Windows, GraphPad Software, CA, USA, www.graphpad.com. Explorative correlation analyses were conducted between all variables. In order to detect location shifts between groups, the non-parametric Mann-Whitney $U$-test for independent samples was used. To determine location shifts within one group at different time points, the Wilcoxon signed-rank test for dependent samples was used. The $X^{2}$-test was used to assess statistically significant differences in sex, aetiology of accident, AO Classification, AIS, the type of paralysis and GCS. Comparison of more than two independent samples was conducted using the Kruskal-Wallis test. Logistic regression models were used to assess the predictive power of variables for improvement in AIS while adjusting for potentially clinically relevant covariates. Model selection for logistic regression was based on $\mathrm{AIC},{ }^{23}$ and clinical relevance of covariates was taken into account. When appropriate, we investigated clinically justifiable interactions and moderation effects. The primary measure for the predictive performance of any logistic regression model was area under the curve (AUC) of the ROC curve. All $P$-values quoted are to be interpreted in a descriptive way as they were not adjusted for multiple testing, and this is an exploratory post hoc analysis.

\section{RESULTS}

This study was designed to be a prospective, explorative study with matched pairs and no randomisation. ${ }^{18}$ Criteria for matching included the patient's sex, age, aetiology and AO classification (Table 2). Patient demographics were documented, and analysis of the entire collective and comparison of groups was performed as in our previous study. ${ }^{16}$

\section{Patients demographics}

In our match-pair analysis, the collective consisted of 30 patients (9 females and 21 males). On average, subjects were $42.03 \pm 17.23$ years of age. Twenty patients were affected by traumatic SCI and serve as the study group (Group $\mathrm{S}=\mathrm{S}$ ). Ten patients had a traumatic injury without neurological impairment and serve as the control group (Group $\mathrm{C}=\mathrm{C}$ ). In the study group, there were 10 patients with AIS improvement (remission) and 10 AIS A grades with no improvement (no remission). There were no lesions of the spinal cord. The clinical characteristics of the entire collective are given in Table 2. 
Table 2 Demographics

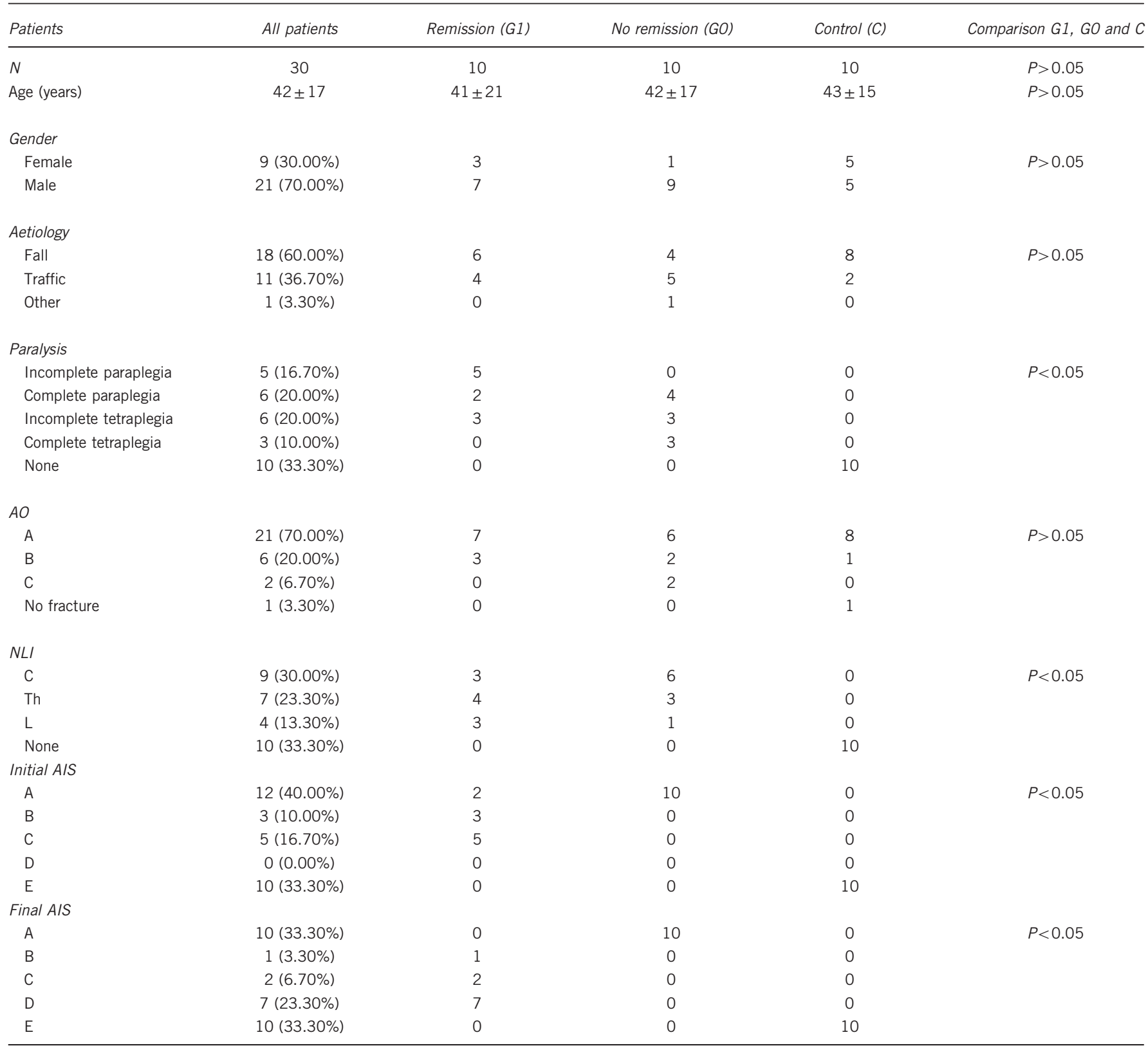

Abbreviations: AO, AO classification; AIS, American Spinal Injury Association (ASIA) Impairment Scale; NLI, neurological level of injury.

Age is expressed as mean years \pm s.d. Neurological remission was defined as improvement in AIS. Comparing GO, G1 and C, $P$-values were analysed with the Kruskal-Wallis test and show differences between GO, G1 and C.

There was no significant difference in the distribution of age, gender, aetiology and $\mathrm{AO}$ classification between patients with and without neurological remission (referred to as G1 and G0 in the following text, respectively). All 20 patients with traumatic SCI (G0 and G1) received surgery (9 ventral 45\%; 11 dorsal 55\%); 14 were treated with spondylodesis $(70.0 \%)$. All 30 patients included in the collective suffered vertebral fractures. The AIS grades at admission and discharge as well as the NLI and the type of paralysis were significantly different in G1 and G0 $(P<0.05)$.

\section{Analysis of the entire patient collective}

For exact MMP concentrations ( $\mathrm{pg} \mathrm{ml}^{-1}$ ) and a graphic comparison of the groups, see Figures 1 and 2. There were no significant differences in cytokine serum levels in regard to gender, age, paralysis, AO classification, aetiology or NLI.

Serum values of MMP-10 and MMP-12 remained undetectable.

\section{Comparison of SCI patients vs control group}

We investigated how MMP values reflect the biochemical processes after SCI by comparing patients with (S) with patients without (C) neurological impairment.

MMP-2. Mean MMP-2 values were higher in S than in C. MMP-2 concentrations of the same first sample fell from $2.95 \mathrm{E}+05$ to $2.43 \mathrm{E}$ $+05 \mathrm{pg} \mathrm{ml}^{-1}$ at $4 \mathrm{~h}$ after trauma. Then they rose continuously to $5.19 \mathrm{E}$ $+05 \mathrm{pg} \mathrm{ml}^{-1}$ at $24 \mathrm{~h}$. Within the first $12 \mathrm{~h}$, MMP-2 in C rose from $1.88 \mathrm{E}+05$ to $2.87 \mathrm{E}+05 \mathrm{pg} \mathrm{ml}^{-1}$ initially and then decreased to $1.95 \mathrm{E}$ 

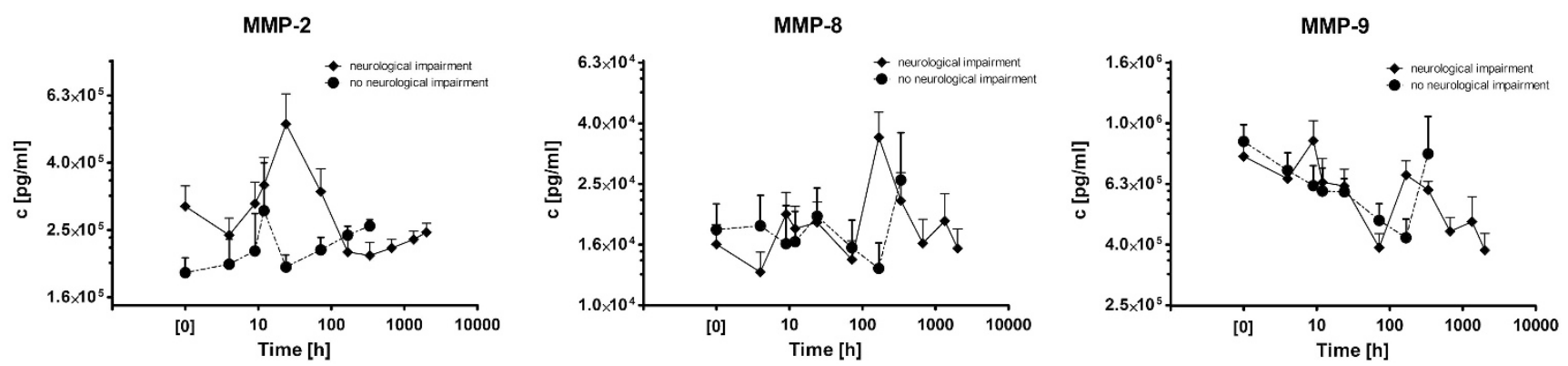

Figure 1 Serum levels of MMP-2, MMP-8 and MMP-9 of all traumatic SCl patients (=study group) and all patients without neurological impairment (=control group), expressed as means \pm s.e.m. The Wilcoxon signed-rank test assessed significant differences from the admission level $(0 \mathrm{~h})$ in the study group, ${ }^{*} P<0.05$ and ${ }^{*} P<0.01$
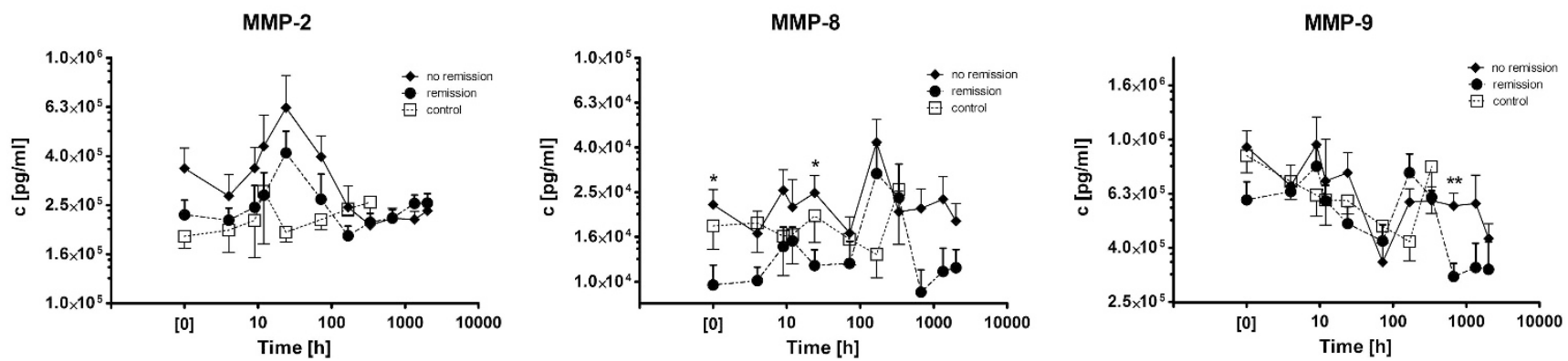

Figure 2 Serum level comparison of all SCl patients with (G1) and without (G0) neurological remission (AIS improvement after 12 weeks), as well as all patients without neurological impairment (=control group). The Mann-Whitney U-test assessed significant differences between G0 and G1 at each particular time point, ${ }^{*} P<0.05$ and ${ }^{* *} P<0.01$.

$+05 \mathrm{pg} \mathrm{ml}^{-1}$. After $24 \mathrm{~h}, \mathrm{~S}$ and $\mathrm{C}$ adjusted to each other at a mean level of $2.41 \mathrm{E}+05 \pm 1.40 \mathrm{E}+04 \mathrm{pg} \mathrm{ml}^{-1}$. No significant difference from admission level has been detected. The Man-Whitney $U$-test assigned no significant difference at every time point.

MMP-8. Mean MMP-8 values were $1.73 \mathrm{E}+04 \pm 1.85 \mathrm{E}+03 \mathrm{pg} \mathrm{ml}^{-1}$ for the first $24 \mathrm{~h}$. MMP-8 concentrations of $\mathrm{S}$ increased after 3 days from $1.42 \mathrm{E}+04$ to $3.58 \mathrm{E}+04 \mathrm{pg} \mathrm{ml}^{-1}$ at 1 week after trauma. In C, MMP-8 fell after 3 days from $1.55 \mathrm{E}+04$ to $1.32 \mathrm{E}+04 \mathrm{pg} \mathrm{ml}^{-1}$ and then rose to $2.59 \mathrm{E}+04 \mathrm{pg} \mathrm{ml}^{-1}$. One week after trauma, we detected a significant difference in the level in $\mathrm{S}$ on admission $(P=0.016)$. The Mann-Whitney $U$-test showed no significant differences at any time point. Afterwards, the levels in both groups adjusted again to each other at a mean level of $1.73 \mathrm{E}+04 \pm 2.17 \mathrm{E}+03 \mathrm{pg} \mathrm{ml}^{-1}$.

MMP-9. Mean MMP-9 values for S and C showed the same pattern for the whole period of time. In $S$, we detected significant differences from the admission level 3 days $(P=0.002), 1 \quad(P=0.011), 2$ $(P=0.017)$ and 3 months $(P=0.011)$ after trauma. The MannWhitney $U$-test assigned no significant difference at any time point. The global MMP-9 trend is decreasing from a mean value of $8.05 \mathrm{E}$ $+05 \pm 7.21 \mathrm{E}+04 \mathrm{pg} \mathrm{ml}^{-1}$ in the beginning to $3.81 \mathrm{E}+05 \pm 5.03 \mathrm{E}$ $+04 \mathrm{pg} \mathrm{ml}^{-1} 3$ months after trauma.

Comparison of patients with neurological remission vs patients without neurological remission

MMP-2. In G0, mean MMP-2 values were mostly higher than those in G1. MMP-2 concentrations in G1 fell initially from 3.55E+05 to $2.74 \mathrm{E}+05 \mathrm{pg} \mathrm{ml}^{-1}$. Then they increased from $4 \mathrm{~h}$ continuously to
$6.27 \mathrm{E}+05 \mathrm{pg} \mathrm{ml}^{-1}$ at $24 \mathrm{~h}$. Within the first $24 \mathrm{~h}, \mathrm{MMP}-2$ increased in G1 from $2.30 \mathrm{E}+05$ to $4.10 \mathrm{E}+05 \mathrm{pg} \mathrm{ml}^{-1}$. Then, both as well as $\mathrm{C}$ stalled at a mean level of $2.41 \mathrm{E}+05 \pm 1.40 \mathrm{E}+04$. Analysis with the Mann-Whitney $U$-test showed no significant differences at any time point during the study.

MMP-8. In G0, mean MMP-8 values were mostly higher than those in G1. G0 and G1 MMP levels were parallel until $24 \mathrm{~h}$ after trauma. Between day 3 and 7, both lines converged before increasing from day 3 to day 7 days and then decreasing to day 14. Afterwards they stabilised at the initial level. The striking differences at admission $(P=0.040)$ and $24 \mathrm{~h}(P=0.036)$ after trauma were significant when analysed with the Mann-Whitney $U$-test (not adjusted for multiple testing).

MMP-9. Starting at $9.39 \mathrm{E}+05 \mathrm{pg} \mathrm{ml}^{-1}$, mean G0 MMP-9 values converged to those of $\mathrm{G} 1$ starting at $5.98 \mathrm{E}+05 \mathrm{pg} \mathrm{ml}^{-1}$ within the first 4 h. Then G0 and G1 showed a similar pattern until 14 days after trauma. G1 decreased significantly $(P=0.035$, Wilcoxon signed-rank test) from $6.12 \mathrm{E}+05$ to $3.11 \mathrm{E}+05 \mathrm{pg} \mathrm{ml}^{-1} 1$ month after trauma and stayed at the same level for the rest of the time span. G0 values remained the same from 14 days to 2 months after trauma and then declined to $4.31 \mathrm{E}+05 \mathrm{pg} \mathrm{ml}^{-1}$. Analysis with the Mann-Whitney $U$ test showed significant differences 1 month $(P=0.007)$ after trauma.

\section{Binary logistic model}

Considering differences of cytokine blood level patterns in the univariate analysis, binary logistic regression models were set up in order to compile a predictive model for the potential of remission. 


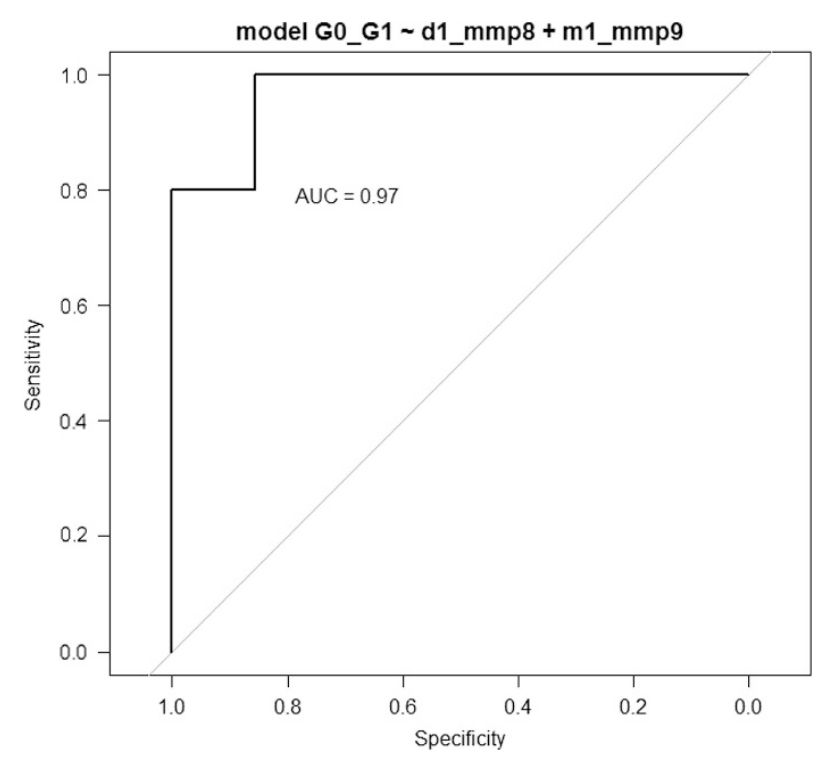

Figure 3 Favoured predictive model including MMP-8 values 1 day and MMP-9 values 1 month after $\mathrm{SCl}$, which enables a prediction of neurological remission in $97 \%$ of cases. Model is based on $n=12$ complete cases.

Patients missing values were excluded from this analysis. Twelve of 20 patients ( 5 with remission, 7 without) were included in the modelling process investigating remission vs no remission. A ROC analysis was performed based on the scores of each model. Considering AIC and AUC values, we preferred MMP-8 value 1 day and MMP-9 value 1 month after injury as predictors. Because of the limited case number a maximum of 2 variables could be included. The ROC curve for the best fitting logistic regression model is shown in Figure 3. The regression model included has an AUC of 0.97 .

\section{DISCUSSION}

This prospective explorative study investigated the expression pattern of MMP-2, MMP-8 and MMP-9 in human peripheral serum throughout the acute, subacute and intermediate phase of traumatic SCI according to a fixed protocol. Because of our large database $(n=115)$ we were able to perform an accurate match-pair analysis with data from previous studies. ${ }^{16,24,25}$ Our goal was to examine MMPs as prognostic markers for neurological remission after SCI and to develop methods of monitoring future therapies. Furthermore, we wanted to prove that there are opportunities for finding a suitable animal model based on our results. The small sample-size limits the informative value of the multivariate statistical analysis. After adjusting for clinically relevant covariates, a significant influence of MMP-8 and MMP-9 might be established and should be further investigated with an enlarged sample size. The AUC indicates good prognostic properties independent of the specific cutoff chosen. However, because of the limited sample size in this study, the exploratory nature of the analysis and several data-driven modelling decisions, a validation of the predictive model is necessary before reaching a final conclusion. We were able to show distinctive expression patterns for MMP-2 and 8, indicating their involvement in the second phase of injury. In addition, significant differences in curves for MMP-8 and 9 distinguished those with neurological remission from those without. The male/female quotient was 2.1:1 and the mean age was $42.03 \pm 16.94$ years as in our previous studies ${ }^{16}$ and within the range of other studies with a sex ratio of $2.5: 1$ to $5.8: 1 .^{26-28}$ We also showed an ongoing shift from young male patients towards a gender balance and older age ${ }^{29}$ As in our previous investigation, we concluded that the number of traffic accidents has decreased because of improvements in the primary prevention sector, ${ }^{30-33}$ as well age increasing as the result of an elevated risk of falls. ${ }^{34,35}$ The present collective consists of a noticeably higher $(60.0 \%)$ number of falls than traffic accidents (36.7\%), in contrast to former observations, which still named traffic accidents as the leading aetiology of SCI. ${ }^{29}$ Numerous animal studies have shown an elevated cytokine serum level after traumatic spinal cord injury. In vitro TNF-alpha has been shown to have cytotoxic effects on oligodendrocytes and consequent damage to white substance. ${ }^{36}$ TNF-alpha receptor-knockout mice, on the other, have shown deteriorated functional regeneration. ${ }^{37}$ These findings underline the complexity of the neuroinflammatory processes and in particular the need for a revised view of neuroinflammatory reactions. Many animalbased studies outlined the relevance of MMP distribution after traumatic injury, especially in regard to their involvement in the secondary phase of injury. ${ }^{38-44}$ They assume a key role of MMPs in the inflammatory and tissue remodelling process. ${ }^{39,41-44}$

\section{MMP-2}

According to Hsu et al., ${ }^{45}$ MMP-2 promotes functional recovery after injury by regulating the formation of a glial scar and white matter sparing and/or axonal plasticity. ${ }^{45}$ In regard to the estimated time span of the remodelling process (within the first 7 days), ${ }^{11}$ these suggestions might correspond partly to our findings. Therefore, we see the differing trend within the first $2 \mathrm{~h}$ after trauma between those with neurological impairment and those without. We conclude that MMP-2 is involved in the post-neurotrauma phase. Surprisingly, we did not see any significant differences between $\mathrm{S}$ and $\mathrm{C}$ nor between G0 and G1. Further studies with an enlarged sample size are needed to clarify our findings. We interpreted the fact that G0 and G1 ran parallel until 1 week as an expression of the severity of the disease and the resulting complexity of the remodelling and recovery process and suggest that a diminished MMP-2 level might indicate functional recovery as hypothesised by Esposito et al. ${ }^{8}$ The MMP-2 analysis suggests that termination has to be settled circa 2 weeks after trauma.

\section{MMP-8}

MMP-8 is of special interest in regard to tissue remodelling after trauma. ${ }^{8,12}$ Our findings revealed a pattern similar to MMP-2, but temporal values showed a different pattern between $12 \mathrm{~h}$ and 7 days after trauma and between those with neurological impairment and those without. We concluded that MMP-8 is involved in the phase of post-neurotrauma. Furthermore, we estimated significantly differing time points regarding functional recovery (at admission $P=0.040$ and after $24 \mathrm{~h} P=0.036$ ). Therefore, patients with an initially lower MMP-8 level as well as a lower level after $24 \mathrm{~h}$ seemed to have had a better chance of neurological improvement. $S$ and $C$ patterns also seemed to be temporally shifted towards each other in MMP-2, as well as in MMP-8. The shift on the timeline in different directions as well as the rigorous blood sampling protocol exclude an artificial effect. In addition, errors based on discordance in clinical sampling might have occurred within the first hours but not after 3 days. Results suggest that neurological impairment affects MMP-2 and MMP-8.

\section{MMP-9}

So far, many studies have investigated the role of MMP-9 in pathophysiological pathways after SCI. ${ }^{38,46-50}$ Although authors have 
provided explicit evidence that MMP-9 is integral to the formation of an inhibitory glial scar and cytoskeleton-mediated astrocyte migration ${ }^{51}$ and indicated that MMP-9 is a key factor involved in the disruption of the endothelial blood-barrier of the spinal cord and subsequent secondary damage after photo thrombotic SCI in rats, ${ }^{39,52}$ we could not find any clear evidence of neurological impairment besides a global trend to decline. Regarding functional improvement, our findings suggest that lower MMP-9 levels provide a higher capacity to recover from neurological impairment (1 month after injury $P=0.007)$. In mice, MMP-9 was shown to promote motor relearning after thoracic spinal cord injury. ${ }^{38}$ In contrast, Piao et al. ${ }^{41}$ found that melatonin improved functional outcome via inhibition of matrix metalloproteinases- 9 after photo thrombotic SCI in rats, suggesting an opposite effect. Furthermore, oxidative stress after SCI induces MMP-9 expression and endothelial injury. ${ }^{49}$ These findings correlate with Yang et al. ${ }^{48}$ showing that hyperbaric oxygen (HBO) reduces SCI-induced spinal cord oedema, stabilising the blood-spinal cord barrier and promoting recovery of neuronal function by downregulating serum levels of IL-6, MMP-2 and MMP-9 and upregulating VEGF. ${ }^{48}$ Apparently the MMP-9 pattern reflects partially the upregulation of MMP-2 within the first $72 \mathrm{~h}$ and MMP- 8 in the time span from $72 \mathrm{~h}$ to 1 month after trauma. In contrast to MMP-8, group C shows a delayed upregulation of MMP-9. We know MMP-9 to be involved in various biochemical processes after traumatic injuries. The present characteristic shows the systemic level and is therefore difficult to interpret. To gain more specific information for MMP-9 regarding neurotrauma, one would need to analyse cerebrospinal fluid. In various approaches, MMP-9 also has been directly or indirectly targeted to influence neurological outcome after SCI. For example, Sulforaphane provides a neuroprotective effect by decreasing mRNA levels of MMP-9. ${ }^{53}$ Increasing NG2+ cell proliferation in the damaged area by targeting MMP-9 resulted in a functional recovery of the spinal cord. ${ }^{40}$

\section{Limitations}

The transferability or conclusions from animal to human models have been shown to hold several deficits; ${ }^{13}$ however, the animal model does have the advantage that is not as limited in the size of the collective. Our explorative prospective study involved a small collective $(n=30)$, and therefore results may not represent local processes clearly. We decided to include different locations and thus differing severities of SCI in G0 and G1. Therefore, in the current study, all patients in G1 have an initial AISA grade A and G0 varies from A to C. This might contribute to differences within the groups and thus disguise patterns in the data. Further studies with an enlarged sample size should address an inter-group (G1 vs G0) comparison with each AISA grade, as the prognosis is often vastly different. Because of the large standard errors to the mean and the small number of patients, the significant differences as described for MMP-8 and MMP-9 must be considered critically. Furthermore, studies must figure out the veracity of the data by increasing the sample size. The temporal changes in metalloproteins after $24 \mathrm{~h}$ (MMP-2), 1 week (MMP-8) and $72 \mathrm{~h}$ (MMP-9) might have a key role in the pathophysiology of SCI and could prove to be good predictive markers. Because of the small sample size we cannot conclude whether they randomly increased at these time points. In order to prove the involvement of MMPs in neuroinflammatory processes, future studies are needed that correlate MMP levels to other established inflammation markers such as interferon- $\gamma$, tumour necrosis factor- $\alpha$, interleukin(IL)-1 $\beta$, IL-6, IL-8, IL-10, and vascular endothelial growth factor. Furthermore, because of missing epitope mapping we can only provide valid evidence for the assay used in the study.

\section{MMP-10}

MMP-10 is known to regulate the lysis of collagen in alternatively activated resident macrophages. Furthermore, MMP-10, which is not a collagenase, promoted an increase in the serum level of MMP- 8 and MMP-13, two metallocollagenases released by macrophages. ${ }^{9}$ Unfortunately, we gained no further information regarding MMP-10 because of the low serum level.

\section{MMP-12}

Wells et al. ${ }^{42}$ obtained data revealing that MMP-12 is destructive and contributes to the development of secondary injury in SCI in mice. This metalloproteinase contributes to the secondary injury process, and in its absence mice recovered better from the insult. ${ }^{42}$ Because of low serum levels we gained no further information about MMP-12.

\section{Predictive modelling}

Although a predictive model stratifying injury severity and predicting neurological outcome might be extremely useful for facilitating the clinical validation of novel treatments in acute human SCI, ${ }^{14}$ findings based on the present modelling process must be considered critical. All $P$-values are unadjusted for multiple testing and should be interpreted descriptively. Because of the small sample size of complete cases $(n=12)$ the predictive value of regression models as well as ROC analysis should be interpreted in an explorative way. Considering the adjusted odds ratios (nearby 1) and their sharp corresponding cis, we have to interpret them in relation to the high serum levels $\left(10^{6}\right.$ and more). Nevertheless, by including the MMP- 8 value after day 1 and the MMP-9 value after 1 month as predictors and by including remission/no remission as a criterion, we gained a predictive model with an $\mathrm{AUC}=0.97$. Besides this statistically impressive result, further studies are needed with a larger collective. Apart from that, this model might be combined with a cost benefit analysis that determined by revealing the ratio of sensitivity/( 1 - specificity) based on the respective clinical question. Thus, an exact cutoff value for sensitivity and specificity might be estimated. On the basis of this information both can be calculated for the model. Therefore, the present study provides key information to developing a reliable SCI score based on biometric data.

\section{Perspective}

So far, our results stage interesting references for the understanding of biochemical processes following SCI. Further studies with an enlarged collective should also focus on patients with an initial AISA A grading and examine their specific cytokine profile regarding the incidence of neurological recovery. Additively, the inclusion of approaches in the radiological assessment still remains very promising sources of information and therefore should also be investigated in the future. In particular, in a setting where a satisfactory clinical examination is not possible, they might provide relevant supplementary information. The current study showed peripheral serum analysis to be a suitable concept for assessing the patient's potential for neurological remission after $\mathrm{tSCI}$. In addition, the present data supply promising approaches for future monitoring concepts for current therapies, as well as animal models. Moreover, the results provide key information for a predictive scoring system for SCI patients. 


\section{CONCLUSIONS}

In the present study, serum level patterns of MMP-2 and MMP-8 showed distinctive patterns for patients with neurological impairment. MMP-8 and MMP-9 patterns showed significant differences regarding functional recovery. Our binary logistic regression model showed that, according to neurological damage, measuring peripheral serum levels can be used to monitor and predict locomotor recovery after SCI. Even though the predictive value of the model in this study must be considered critically, results provided a basis for further approaches investigating standardized monitoring as well as prognostic tracking techniques and scoring.

\section{DATA ARCHIVING}

There were no data to deposit.

\section{CONFLICT OF INTEREST}

The authors declare no conflict of interest.

\section{ACKNOWLEDGEMENTS}

We thank Martina Kutsche-Bauer for performing the Luminex assays.

Statistical consulting was provided by the Institute for Medical Biometrics and Information Technology at the University of Heidelberg, Germany.

1 Boakye M, Leigh BC, Skelly AC. Quality of life in persons with spinal cord injury: comparisons with other populations. J Neurosurg Spine 2012; 17: 29-37.

2 Rowland JW, Hawryluk GW, Kwon B, Fehlings MG. Current status of acute spinal cord injury pathophysiology and emerging therapies: promise on the horizon. Neurosurg Focus 2008; 25: E2.

3 Devivo MJ. Epidemiology of traumatic spinal cord injury: trends and future implications. Spinal Cord 2012; 50: 365-372.

4 Ito Y, Sugimoto Y, Tomioka M, Kai N, Tanaka M. Does high dose methylprednisolone sodium succinate really improve neurological status in patient with acute cervical cord injury?: a prospective study about neurological recovery and early complications. Spine (Phila Pa 1976) 2009; 34: 2121-2124.

5 Kwon BK, Okon EB, Plunet W, Baptiste D, Fouad K, Hillyer J et al. A systematic review of directly applied biologic therapies for acute spinal cord injury. J Neurotrauma 2011; 28: 1589-1610.

6 Evaniew N, Belley-Cote EP, Fallah N, Noonan VK, Rivers CS, Dvorak MF. Methylprednisolone for the treatment of patients with acute spinal cord injuries: a systematic review and meta-analysis. J Neurotrauma 2015; 33: 468-481.

7 Walters BC, Hadley MN, Hurlbert RJ, Aarabi B, Dhall SS, Gelb DE et al. Guidelines for the management of acute cervical spine and spinal cord injuries: 2013 update. Neurosurgery 2013; 60: 82-91.

8 Esposito E, Genovese T, Caminiti R, Bramanti P, Meli R, Cuzzocrea S. Melatonin regulates matrix metalloproteinases after traumatic experimental spinal cord injury. J Pineal Res 2008; 45: 149-156.

9 Rohani MG, McMahan RS, Razumova MV, Hertz AL, Cieslewicz M, Pun SH et al. MMP-10 regulates collagenolytic activity of alternatively activated resident macrophages. J Invest Dermatol 2015; 135: 2377-2384.

10 Kwon BK, Casha S, Hurlbert RJ, Yong VW. Inflammatory and structural biomarkers in acute traumatic spinal cord injury. Clin Chem Lab Med 2011; 49: 425-433.

11 Yokobori S, Zhang Z, Moghieb A, Mondello S, Gajavelli S, Dietrich WD et al. Acute diagnostic biomarkers for spinal cord injury: review of the literature and preliminary research report. World Neurosurg 2015; 83: 867-878.

12 Zhang $\mathrm{H}$, Chang M, Hansen CN, Basso DM, Noble-Haeusslein LJ. Role of matrix metalloproteinases and therapeutic benefits of their inhibition in spinal cord injury. Neurotherapeutics 2011; 8: 206-220.

13 Kwon BK, Streijger F, Hill CE, Anderson AJ, Bacon M, Beattie MS et al. Large animal and primate models of spinal cord injury for the testing of novel therapies. Exp Neurol 2015; 269: 154-168.

14 Kwon BK, Stammers AM, Belanger LM, Bernardo A, Chan D, Bishop CM et al. Cerebrospinal fluid inflammatory cytokines and biomarkers of injury severity in acute human spinal cord injury. J Neurotrauma 2010; 27: 669-682.

15 Moghaddam A, Breier L, Haubruck P, Bender D, Biglari B, Wentzensen A et al. Nonunions treated with bone morphogenic protein 7: introducing the quantitative measurement of human serum cytokine levels as promising tool in evaluation of adjunct non-union therapy. J Inflamm (Lond) 2016; 13: 3.

16 Moghaddam A, Child C, Bruckner T, Gerner HJ, Daniel V, Biglari B. Posttraumatic inflammation as a key to neuroregeneration after traumatic spinal cord injury. Int J Mol Sci 2015; 16: 7900-7916.
17 Moghaddam A, Zimmermann G, Hammer K, Bruckner T, Grutzner PA, von Recum J. Cigarette smoking influences the clinical and occupational outcome of patients with tibial shaft fractures. Injury 2011; 42: 1435-1442.

18 Moghaddam A, Muller U, Roth HJ, Wentzensen A, Grutzner PA, Zimmermann G. TRACP $5 \mathrm{~b}$ and CTX as osteological markers of delayed fracture healing. Injury 2011; 42: 758-764.

19 Kirshblum SC, Burns SP, Biering-Sorensen F, Donovan W, Graves DE, Jha A et al. International standards for neurological classification of spinal cord injury (revised 2011). J Spinal Cord Med 2011; 34: 535-546.

20 Marino RJ, Ditunno JF Jr, Donovan WH, Maynard F Jr Neurologic recovery after traumatic spinal cord injury: data from the Model Spinal Cord Injury Systems. Arch Phys Med Rehabil 1999; 80: 1391-1396.

21 R Development Core Team (2015). R: A Language and Environment for Statistical Computing. Vienna, Austria, R Foundation for Statistical Computing.

22 Robin X, Turck N, Hainard A, Tiberti N, Lisacek F, Sanchez JC et al. pROC: an opensource package for $\mathrm{R}$ and $\mathrm{S}+$ to analyze and compare ROC curves. BMC Bioinformatics 2011; 12: 77.

23 Akaike $\mathrm{H}$. Information Theory and an Extension of the Maximum Likelihood Principle. Selected Papers of Hirotugu Akaike. (eds. Parzen E, Tanabe K, Kitagawa G) New York, NY, Springer New York: 199-213 (1998).

24 Biglari B, Buchler A, Swing T, Biehl E, Roth HJ, Bruckner T et al. Increase in soluble CD95L during subacute phases after human spinal cord injury: a potential therapeutic target. Spinal Cord 2013; 51: 183-187.

25 Biglari B, Buchler A, Swing T, Child C, Biehl E, Reitzel T et al. Serum SCD95L concentration in patients with spinal cord injury. J Int Med Res 2015; 43: 250-256.

26 Osterthun R, Post MW, van Asbeck FW. Characteristics, length of stay and functional outcome of patients with spinal cord injury in Dutch and Flemish rehabilitation centres. Spinal Cord 2009; 47: 339-344.

27 Milicevic S, Bukumiric Z, Nikolic AK, Babovic R, Jankovic S. Demographic characteristics and functional outcomes in patients with traumatic and nontraumatic spinal cord injuries. Vojnosanit Preg/ 2012; 69: 1061-1066.

28 Wyndaele M, Wyndaele JJ. Incidence, prevalence and epidemiology of spinal cord injury: what learns a worldwide literature survey? Spinal Cord 2006; 44: 523-529.

29 Jackson AB, Dijkers M, Devivo MJ, Poczatek RB. A demographic profile of new traumatic spinal cord injuries: change and stability over 30 years. Arch Phys Med Rehabil 2004; 85: 1740-1748.

30 Cummins JS, Koval KJ, Cantu RV, Spratt KF. Do seat belts and air bags reduce mortality and injury severity after car accidents? Am J Orthop (Belle Mead, NJ) 2011; 40: E26-E29.

31 Smith JL, Ackerman LL. Management of cervical spine injuries in young children: lessons learned. J Neurosurg Pediatr 2009; 4: 64-73.

32 Weninger $\mathrm{P}$, Hertz $\mathrm{H}$. Factors influencing the injury pattern and injury severity after high speed motor vehicle accident-a retrospective study. Resuscitation 2007; 75: 35-41.

33 Richter M, Thermann H, Wippermann B, Otte D, Schratt HE, Tscherne H. Foot fractures in restrained front seat car occupants: a long-term study over twenty-three years. J Orthop Trauma 2001; 15: 287-293.

34 Kaminska MS, Brodowski J, Karakiewicz B. Fall risk factors in community-dwelling elderly depending on their physical function, cognitive status and symptoms of depression. Int J Environ Res Public Health 2015; 12: 3406-3416.

35 Chen Y, Tang Y, Allen V, DeVivo MJ. Aging and spinal cord injury: external causes of injury and implications for prevention. Top Spinal Cord Inj Rehabil 2015; 21: 218-226.

36 Merrill JE, Ignarro LJ, Sherman MP, Melinek J, Lane TE. Microglial cell cytotoxicity of oligodendrocytes is mediated through nitric oxide. J Immunol 1993; 151: 2132-2141.

37 Kim GM, Xu J, Song SK, Yan P, Ku G, Xu XM et al. Tumor necrosis factor receptor deletion reduces nuclear factor-kappaB activation, cellular inhibitor of apoptosis protein 2 expression, and functional recovery after traumatic spinal cord injury. J Neurosci $2001 ; 21: 6617-6625$.

38 Hansen CN, Fisher LC, Deibert RJ, Jakeman LB, Zhang H, Noble-Haeusslein L et al. Elevated MMP-9 in the lumbar cord early after thoracic spinal cord injury impedes motor relearning in mice. J Neurosci 2013; 33: 13101-13111.

39 Jang JW, Lee JK, Kim SH. Activation of matrix metalloproteinases-9 after photothrombotic spinal cord injury model in rats. J Korean Neurosurg Soc 2011; 50: 288-292.

40 Liu H, Shubayev VI. Matrix metalloproteinase-9 controls proliferation of NG2+ progenitor cells immediately after spinal cord injury. Exp Neurol 2011; 231: 236-246.

41 Piao MS, Lee JK, Jang JW, Hur H, Lee SS, Xiao L et al. Melatonin improves functional outcome via inhibition of matrix metalloproteinases-9 after photothrombotic spinal cord injury in rats. Acta Neurochir (Wien) 2014; 156: 2173-2182.

42 Wells JE, Rice TK, Nuttall RK, Edwards DR, Zekki H, Rivest S et al. An adverse role for matrix metalloproteinase 12 after spinal cord injury in mice. J Neurosci 2003; 23: 10107-10115.

43 Zhang $\mathrm{H}$, Trivedi A, Lee JU, Lohela M, Lee SM, Fandel TM et al. Matrix metalloproteinase-9 and stromal cell-derived factor-1 act synergistically to support migration of blood-borne monocytes into the injured spinal cord. J Neurosci 2011; 31: $15894-15903$

44 Zhou Y, Cui Z, Xia X, Liu C, Zhu X, Cao J et al. Matrix metalloproteinase-1 (MMP-1) expression in rat spinal cord injury model. Cell Mol Neurobiol 2014; 34: 1151-1163.

45 Hsu JY, McKeon R, Goussev S, Werb Z, Lee JU, Trivedi A et al. Matrix metalloproteinase-2 facilitates wound healing events that promote functional recovery after spinal cord injury. J Neurosci 2006; 26: 9841-9850. 
46 Lee JY, Choi HY, Na WH, Ju BG, Yune TY. 17beta-estradiol inhibits MMP-9 and SUR1/ TrpM4 expression and activation and thereby attenuates BSCB disruption/hemorrhage after spinal cord injury in male rats. Endocrinology 2015; 156: 1838-1850.

47 Li XQ, Cao XZ, Wang J, Fang B, Tan WF, Ma H. Sevoflurane preconditioning ameliorates neuronal deficits by inhibiting microglial MMP-9 expression after spinal cord ischemia/ reperfusion in rats. Mol Brain 2014; 7: 69.

48 Yang J, Wang G, Gao C, Shao G, Kang N. Effects of hyperbaric oxygen on MMP-2 and MMP-9 expression and spinal cord edema after spinal cord injury. Life Sci 2013; 93: 1033-1038.

49 Yu F, Kamada H, Niizuma K, Endo H, Chan PH. Induction of mmp-9 expression and endothelial injury by oxidative stress after spinal cord injury. J Neurotrauma 2008; 25: 184-195.
50 Zhang P, Ma X. Effect of rutin on spinal cord injury through inhibition of the expression of MIP-2 and activation of MMP-9, and downregulation of Akt phosphorylation. Mol Med Rep 2015; 12: 7554-7560.

51 Hsu JY, Bourguignon LY, Adams CM, Peyrollier K, Zhang H, Fandel T et al. Matrix metalloproteinase-9 facilitates glial scar formation in the injured spinal cord. J Neurosci 2008; 28: 13467-13477.

52 Austin JW, Afshar M, Fehlings MG. The relationship between localized subarachnoid inflammation and parenchymal pathophysiology after spinal cord injury. J Neurotrauma 2012; 29: 1838-1849.

53 Benedict AL, Mountney A, Hurtado A, Bryan KE, Schnaar RL, Dinkova-Kostova AT et al. Neuroprotective effects of sulforaphane after contusive spinal cord injury. J Neurotrauma 2012; 29: 2576-2586. 